\title{
Inclusión de ajo (Allium sativum) y cebolla (Allium cepa) en la dieta sobre los parámetros productivos en pollos de engorde
}

\section{Addition of garlic (Allium sativum) and onion (Allium cepa) in the diet on productive parameters in broiler chickens}

\author{
Carlomagno Ronald Velásquez Vergara ${ }^{l}$, Jaime Fernando Vega-Vilca ${ }^{l}$, Hilario Noberto Pujada Abad $^{l}$ y \\ Félix Esteban Airahuacho Bautista ${ }^{l}$
}

${ }^{1}$ Universidad Nacional José Faustino Sánchez Carrión, Perú.

RESUMEN

El uso intensivo de los antibióticos promotores del crecimiento (APC) ha originado resistencia bacteriana con efectos perjudiciales para la salud, ante esta situación es necesario buscar productos alternativos, como los productos fitogénicos. El objetivo de la investigación fue evaluar el efecto de la harina de ajo y cebolla en la dieta sobre el rendimiento productivo y uniformidad de lote en pollos de engorde. Se utilizaron 150 pollos machos de carne de la línea Cobb 500.

Las aves se distribuyeron al azar en tres grupos con cinco replicaciones y cada replicación con diez pollos. Los tratamientos fueron: T0: Control negativo, sin APC; T1: Control positivo, con APC (Enramicina 8\%); T2: Harina de ajo 0,75\% + cebolla $0,75 \%$. Las variables de rendimiento productivo fueron: peso vivo, consumo de alimento, conversión alimenticia y uniformidad de lote. Se utilizó el análisis de varianza de un diseño completamente al azar (DCA) y la prueba de Tukey para evaluar las variables estudiadas. No se encontraron diferencias significativas $(\mathrm{P}>0.05)$ en el rendimiento productivo, al comparar el tratamiento de harina de ajo y cebolla (T2) con los tratamientos sin APC (T1) y con APC (T2); la uniformidad de lote fue elevada, sin mostrar diferencias $(\mathrm{P}>0,05)$ entre los tratamientos. La harina de ajo y cebolla podría constituir una alternativa de uso en la dieta alimenticia de pollos de engorde.

Palabras clave: harina de ajo, cebolla, rendimiento productivo, pollos de engorde.

\section{AbSTRACT}

The intensive use of growth-promoting antibiotics (APC) has led to bacterial resistance with harmful effects on health, in this situation it is necessary to look for alternative products, such as phytlogenic products. The objective of the research was to evaluate the effect of garlic and onion flour in the diet on productive performance and batch uniformity in broilers. 150 male beef chickens of the Cobb 500 line were used.

The broilers were randomly distributed into three groups with five replications and each replication with ten chickens. The treatments were: T0: Negative control, without APC; T1: Positive control, with APC (Enramycin 8\%); T2: Garlic flour 0.75\% 
+ onion $0.75 \%$. The productive performance variables were: live weight, feed consumption, feed conversion and batch uniformity. The analysis of variance of a completely randomized design (DCA) and the Tukey test were used to evaluate the variables studied. No significant differences $(\mathrm{P}>0.05)$ were found in the productive performance, when comparing the treatment of garlic and onion flour (T2) with the treatments without APC (T0) and with APC (T1); batch uniformity was high, without showing differences $(\mathrm{P}>0.05)$ between treatments. Garlic and onion flour could be an alternative for use in the diet of broilers.

Keywords: garlic flour, onion, productive performance, broilers.

\section{INTRODUCCIÓN}

$\mathrm{E}^{1}$ uso intensivo de los antimicrobianos en las granjas avícolas ha originado la aparición de cepas bacterianas resistentes generando un problema de salud pública (Roskam et al., 2020). Ante esta situación, es necesario buscar productos alternativos, como los productos fitogénicos que coadyuven en la prevención y tratamiento de las enfermedades infecciosas, no sólo eliminando al agente patógeno sino, además, estimulando el buen funcionamiento del sistema inmunológico, para de esta manera neutralizar a estos patógenos y evitar desde un primer momento se multiplique dentro del organismo animal y produzca la enfermedad.

Las plantas medicinales tienen diversos mecanismos de acción para eliminar a los microorganismos patógenos. Algunos fitoconstituyentes como los alcaloides y lactonas esteroidales incrementan la fagocitosis por parte de los macrófagos y neutrófilos, de esta manera se mejora las defensas del hospedero (Sugiharto y Ranjitkar, 2019); los flavonides, esteroides, B-carotenos, antocianinas, antraquinonas, glucósidos, cumarina y alcaloides estimulan una mayor formación de anticuerpos y tienen propiedades antimicrobianas, incluso son efectivas contra muchas cepas de bacterias resistentes a los antibióticos (Sharma et al., 2014). La acción inmunomoduladora de las plantas medicinales en el hospedero se realiza por diversos mecanismos como, la regulación de las citosinas para controlar la inflamación; proliferación de linfocitos $\beta$ formadoras de anticuerpos; estimulación de la inmunidad celular, incremento de la fagocitosis en los macrófagos; muerte bacteriana por daño a la membrana celular e inhibición de la patogénesis bacteriana, por regulación negativa de la síntesis de enzimas específicas (Sugiharto y Ranjitkar, 2019).

El ajo (Allium sativum) es una planta medicinal que se utiliza desde tiempos antiguos en la prevención y tratamiento de una variedad de enfermedades (Adibmoradi et al., 2006). Sus principales componentes bioactivos, como la alicina, tienen un efecto antimicrobiano, antifúngico, estimulante del sistema digestivo animal, antioxidante, anticoccidial, aumenta la producción de enzimas digestivas y mejora la utilización de productos digestivos optimizando las funciones hepáticas (Corzo-Martínez et al., 2007; Eid y Iraqui, 2014) menciona, además, que tienen un efecto estimulante de la respuesta inmunológica en pollos (Elmowalid, 2019).

La cebolla (Alliium cepa) pertenece a la familia Liliaceae. Los bulbos de cebolla tienen numerosos compuestos de azufre, flavonoides y ácidos fenólicos con eficacia antibacteriana, antioxidante e hipolipidémica probada (An, et al., 2015), que mejora la composición de la flora intestinal, estimula el desarrollo del pollo y favorece el crecimiento de los órganos linfoides como el bazo y la bolsa de Fabricio (Mulugeta, 2018).

Existen pocos estudios sobre la aplicación de las propiedades medicinales del ajo y cebolla en los animales de granja. A nivel nacional no se cuenta con estudios que demuestren las bondades medicinales de estos productos en aves. El objetivo de la investigación fue evaluar el efecto de la harina de ajo y cebolla en la dieta alimenticia sobre el rendimiento productivo y la uniformidad de lote en pollos de engorde.

\section{MATERIALES Y MÉTODOS}

El trabajo de investigación se realizó en la granja experimental de aves de la Escuela Profesional de Ingeniería Zootécnica, ubicada en la zona sur oeste de la ciudad universitaria, distrito de Huacho, departamento de Lima, a 27 metros sobre el nivel del mar; durante los meses de diciembre 2020 a enero 2021. La temperatura y humedad en el galpón osciló entre $19-26{ }^{\circ} \mathrm{C}$ y $60-70 \%$ respectivamente, de acuerdo al momento de la crianza.

Se trabajó en un galpón con piso de cemento, enmallado en los alrededores para evitar ingreso de animales silvestres y corrales de $1 \mathrm{~m} 2$ con cama de viruta para cada unidad experimental. Se utilizaron 150 pollos machos de carne de la línea Cobb 500. Las aves se distribuyeron al azar en tres tratamientos; cada tratamiento con cinco replicaciones y cada replicación con diez pollos. Los pollos fueron vacunados al día de nacido contra las enfermedades de Newcastle (NC), Marek (EM) y Bronquitis infecciosa (BI). En el día 12 se aplicó la segunda dosis de NC y BI.

El alimento, de presentación física en polvo, se suministró en bandejas circulares hasta los 3 días, luego se cambió a tolvas. El agua fue suministrada en bebederos tipo tolva (etapa inicial) y bebederos chupones (etapa de crecimiento y acabado). La altura de las cortinas se graduó de acuerdo al crecimiento del pollo.

La harina de ajo y cebolla fue adquirida de una empresa comercializadora de dichos productos. El análisis químico proximal del ajo y cebolla se realizaron en el laboratorio de evaluación nutricional de la Universidad Nacional Agraria la Molina, Lima-Perú, siguiendo la metodología de la AOAC, (2005). Los resultados mostrados en la Tabla 1, fueron incorporados en la base de datos para la formulación de las dietas experimentales. 
Tabla 1.

ISSNe: 2617-9156

Análisis químico proximal de la harina de ajo y cebolla.

\begin{tabular}{ccc} 
Composición & Harina de cebolla & Harina de ajo \\
\hline Humedad, \% & 18,55 & 12,19 \\
Proteína total (N x 6,25), \% & 10,34 & 10,03 \\
Grasa, \% & 0,51 & 0,53 \\
Fibra cruda, \% & 4,97 & 0,88 \\
Ceniza, \% & 3,10 & 1,62 \\
ELN ${ }^{1} \%$ & 2,53 & 74,75 \\
\hline
\end{tabular}

$1 \mathrm{ELN}=$ Extracto Libre de Nitrógeno.

Se elaboraron dietas para cada etapa productiva del pollo de engorde, considerando la etapa inicial de 1 a 7 días, etapa de crecimiento de 8 a 21 días y etapa de acabado de 22 a 42 días. Las dietas experimentales o tratamientos (Tabla 2) se formularon de acuerdo a las recomendaciones nutricionales de Rostagno et al. (2017) y fueron: T0: Control negativo, sin APC; T1: Control positivo, con APC (Enramicina 8\%); T2: Harina de ajo $0,75 \%$ + cebolla $0,75 \%$.

Tabla 2.

Dietas alimenticias suministradas según etapa de desarrollo, para cada tratamiento (sin APC, con APC, ajos+ cebolla) en pollos de engorde.

\begin{tabular}{|c|c|c|c|c|c|c|c|c|c|}
\hline \multirow{2}{*}{ Insumos } & \multicolumn{3}{|c|}{ Inicio (0-7 días) } & \multicolumn{3}{|c|}{ Crecimiento (8-21 días) } & \multicolumn{3}{|c|}{ Acabado (22-42 días) } \\
\hline & $\mathbf{T}_{0}{ }^{1}$ & $\mathbf{T}_{1}{ }^{2}$ & $\mathbf{T}_{2}{ }^{3}$ & $\mathbf{T}_{\mathbf{0}}$ & $\mathbf{T}_{1}$ & $\mathbf{T}_{2}$ & $\mathbf{T}_{\mathbf{0}}$ & $\mathbf{T}_{1}$ & $\mathbf{T}_{2}$ \\
\hline Maíz & 64,15 & 64,08 & 62,65 & 60,89 & 60,83 & 59,39 & 63,91 & 63,85 & 62,43 \\
\hline Torta de soya 45 & 17,90 & 17,90 & 17,50 & 25,30 & 25,30 & 24,80 & 27,00 & 27,30 & 27,20 \\
\hline Soya integral extruida & 13,60 & 13,60 & 14,00 & 5,20 & 5,20 & 5,70 & 0,00 & 0,00 & 0,00 \\
\hline Harina de cebolla & 0,00 & 0,00 & 0,75 & 0,00 & 0,00 & 0,75 & 0,00 & 0,00 & 0,75 \\
\hline Harina de ajo & 0,00 & 0,00 & 0,75 & 0,00 & 0,00 & 0,75 & 0,00 & 0,00 & 0,75 \\
\hline Aceite de soya & 0.00 & 0,00 & 0,00 & 4,00 & 4,00 & 4,00 & 4,44 & 4,44 & 4,52 \\
\hline Carbonato de calcio & 0.87 & 0,87 & 0,87 & 0,60 & 0,60 & 0,60 & 0,53 & 0,53 & 0,53 \\
\hline Fosfato bicálcico & 1,52 & 1,52 & 1,52 & 2,20 & 2,20 & 2,20 & 1,98 & 1,98 & 1,98 \\
\hline DL metionina 99 & 0,37 & 0,37 & 0,37 & 0,33 & 0,33 & 0,33 & 0,34 & 0,34 & 0,34 \\
\hline Lisina 78 & 0,42 & 0,42 & 0,42 & 0,31 & 0,31 & 0,31 & 0,35 & 0,35 & 0,36 \\
\hline Treonina & 0,16 & 0,16 & 0,16 & 0,13 & 0,13 & 0,13 & 0,13 & 0,13 & 0,13 \\
\hline Sal & 0,32 & 0,32 & 0,32 & 0,32 & 0,32 & 0,32 & 0,30 & 0,30 & 0,29 \\
\hline $\mathrm{APC} *$ & 0,00 & 0,015 & 0,00 & 0,00 & 0,015 & 0,00 & 0,00 & 0,015 & 0,00 \\
\hline Coccidiostato & 0,00 & 0,050 & 0,00 & 0,00 & 0,050 & 0,00 & 0,00 & 0,050 & 0,00 \\
\hline Aditivos $* *$ & 0,695 & 0,695 & 0,695 & 0,720 & 0,720 & 0,720 & 0,720 & 0,720 & 0,720 \\
\hline \multicolumn{10}{|l|}{ Contenido nutricional } \\
\hline Energía Metabol., Mcal/kg & 3,00 & 3,00 & 3,00 & 3,05 & 3,05 & 3,05 & 3,05 & 3,05 & 3,05 \\
\hline Proteína bruta, $\%$ & 19,00 & 19,00 & 19,00 & 18,50 & 18,50 & 18,50 & 17,50 & 17,50 & 17,50 \\
\hline Lisina digest., \% & 1,15 & 1,15 & 1,15 & 1,07 & 1,07 & 1,07 & 1,02 & 1,02 & 1,02 \\
\hline Met + Cis digest., $\%$ & 0,87 & 0,87 & 0,87 & 0,83 & 0,83 & 0,83 & 0,80 & 0,80 & 0,80 \\
\hline Treonina digest., $\%$ & 0,75 & 0,75 & 0,75 & 0,70 & 0,70 & 0,70 & 0,66 & 0,66 & 0,66 \\
\hline Triptófano digest., \% & 0,18 & 0,18 & 0,18 & 0,18 & 0,18 & 0,18 & 0,16 & 0,16 & 0,16 \\
\hline Grasa, $\%$ & 5,30 & 5,30 & 5,34 & 7,60 & 7,70 & 7,64 & 7,20 & 7,20 & 7,24 \\
\hline Fibra, \% & 3,28 & 3,28 & 3,29 & 3,03 & 3,00 & 3,03 & 3,08 & 3,08 & 3,09 \\
\hline Calcio, $\%$ & 0,90 & 0,90 & 0,90 & 0,86 & 0,86 & 0,86 & 0,76 & 0,76 & 0,76 \\
\hline Fósforo digest., \% & 0,45 & 0,45 & 0,45 & 0,43 & 0,43 & 0,43 & 0,38 & 0,38 & 0,38 \\
\hline Sodio, $\%$ & 0,18 & 0,18 & 0,18 & 0,18 & 0,18 & 0,18 & 0,18 & 0,18 & 0,18 \\
\hline Balance electrolítico, $\mathrm{mEq} / \mathrm{kg}$ & 227 & 227 & 224 & 219 & 219 & 218 & 228 & 228 & 225 \\
\hline
\end{tabular}

${ }^{1} \mathrm{~T}_{0}$ : Control negativo, sin APC

${ }^{2} \mathrm{~T}_{1}$ : Control positivo: con APC

${ }^{3} \mathrm{~T}_{2}$ : Harina de ajo+ cebolla $(0,75 \%+0,75 \%$

* $\mathrm{APC}=$ Antibiótico promotor de crecimiento (Enramicina $8 \%$ )

** $0,1 \%$ cloruro de colina $60 \% ; 0,2 \%$ bicarbonato de sodio; $0,02 \%$ vitamina $\mathrm{C} ; 0,1 \%$ antifúngico; $0,2 \%$ secuestrante de micotoxinas; $01 \%$ premezcla de vitaminas y minerales. 
Las variables dependientes evaluadas fueron:

Peso vivo, $P V(g)=\frac{\text { Suma de peso de los pollos }(g)}{\text { Número de pollos pesados }}$

Alimento consumido, $C A L=\frac{\text { Consumo de alimento total }(g)}{\text { Número de pollos }}$

Conversión alimenticia, $\mathrm{CA}=\frac{\text { Consumo de alimento }(g)}{\text { Peso vivo }(g)}$

Uniformidad de lote $(\%)=100-[$ desviación estándar $(\mathrm{g}) /$ Promedio peso corporal $(g) x$ 100]

La uniformidad del lote se determinó de acuerdo a lo propuesto por Ojediran et al. (2017)

Los datos se procesaron en el programa Minitab v. 18 y se presentaron en tablas mostrando el promedio, la desviación estándar y el coeficiente de variación. El análisis de varianza (ANOVA) de un diseño completo al azar (DCA) se utilizó para determinar diferencias entre los tratamientos y la prueba de Tukey permitió identificar diferencias entre tratamientos.

\section{RESUltado}

La Tabla 3 muestra que no hubo diferencias significativas $(\mathrm{P}>0,05)$ entre los tratamientos en relación al PV, CAL y CA. El rendimiento productivo se encuentra acorde a los estándares establecidos por la línea Cobb 500 (2018b).

Tabla 3.

Efecto de los tratamientos con APC y harina de ajo + cebolla en el rendimiento productivo en pollos de engorde.

\begin{tabular}{ccccc}
\hline \multirow{2}{*}{ Fase } & Variable & To: Control & T1: APC & T2: Ajo + Cebolla \\
& Promedio \pm d.e. & Promedio \pm d.e. & Promedio \pm d.e. \\
\hline \multirow{2}{*}{7 días } & Peso corporal $(\mathrm{g})$ & $185,96 \pm 9,55$ & $188,04 \pm 11,02$ & $189,04 \pm 6,06$ \\
& Consumo alimento $(\mathrm{g})$ & $146,78 \pm 6,23$ & $141,08 \pm 12,72$ & $151,72 \pm 4,72$ \\
& Conversión alimenticia & $1,04 \pm 0,06$ & $0,99 \pm 0,07$ & $1,05 \pm 0,03$ \\
\hline \multirow{2}{*}{21 días } & Peso corporal $(\mathrm{g})$ & $1056,90 \pm 32,30$ & $1080,50 \pm 67,60$ & $1051,70 \pm 39,40$ \\
& Consumo alimento $(\mathrm{g})$ & $1278,30 \pm 38,40$ & $1224,60 \pm 50,00$ & $1282,20 \pm 40,00$ \\
& Conversión alimenticia & $1,26 \pm 0,03$ & $1,19 \pm 0,12$ & $1,27 \pm 0,08$ \\
\hline \multirow{2}{*}{42 días } & Peso corporal $(\mathrm{g})$ & $3034,50 \pm 149,70$ & $3036,80 \pm 38,20$ & $3063,30 \pm 89,00$ \\
& Consumo alimento $(\mathrm{g})$ & $5024,90 \pm 183,50$ & $5071,80 \pm 197,30$ & $5128,00 \pm 279,00$ \\
\hline
\end{tabular}

${ }^{1}$ APC: antibiótico promotor del crecimiento (Enramicina $8 \%$ ).

${ }^{2}$ Harina de ajo + cebolla $(0,75+0,75 \%)$.

La Tabla 4 muestra el porcentaje de uniformidad del lote durante las tres fases de crianza. No se encontraron diferencias entre los tratamientos $(\mathrm{P}>0,05)$, se caracterizó por ser elevada y con baja variabilidad en las etapas de inicio, crecimiento y engorde. Los resultados se encuentran por encima de los estándares recomendados por la guía de crianza y manejo del pollo de engorde (Cobb 500, 2018a).

\section{Tabla 4.}

Porcentaje de uniformidad del peso vivo, según los tratamientos ( $\sin$ APC, con APC y harina de ajo + cebolla) en pollos de engorde.

\begin{tabular}{ccccccc}
\hline & \multicolumn{2}{c}{ To: $\sin \mathbf{A P C}^{\mathbf{1}}$} & \multicolumn{2}{c}{ T1: con APC } & \multicolumn{2}{c}{ T2: $_{\mathbf{2}}$ ajo+cebolla } \\
\cline { 2 - 6 } Fase (días) & Promedio \pm d.e. & $\mathbf{C V}^{\mathbf{3}}$ & Promedio \pm d.e. & C.V. & Promedio \pm d.e. & C.V. \\
\hline & & & & & & \\
7 & $91,75 \pm 1,84$ & 2,00 & $89,57 \pm 5,61$ & 6,26 & $92,86 \pm 1,73$ & 1,86 \\
21 & $92,78 \pm 2,21$ & 2,38 & $93,72 \pm 1,19$ & 1,26 & $93,37 \pm 0,94$ & 1,00 \\
42 & $93,01 \pm 1,63$ & 1,75 & $93,66 \pm 2,08$ & 2,22 & $91,62 \pm 1,89$ & 2,06 \\
\hline
\end{tabular}

\footnotetext{
${ }^{1} \mathrm{APC}=$ Enramicina $8 \%$

${ }^{2}$ Harina de ajo $0,75 \%$ + cebolla $0,75 \%$

${ }^{3} \mathrm{CV}$ : coeficiente de variación
} 
ISSNe: 2617-9156

\section{Discusión}

Los resultados logrados con el tratamiento de harina de ajo + cebolla $(0,75 \%+0,75 \%)$ fueron similares a los obtenidos por algunos autores como, An et al. (2015) quienes observaron que los pollitos alimentados con $0,3 \%$ ó $0,5 \%$ de extracto de cebolla mostraron un PV similar al del grupo control medicado; Abudabos et al. (2018) no encontraron diferencias en el PV, CAL y CA al comparar tratamientos con extractos medicinales y APC. Otros autores si encontraron respuesta favorable con el uso de ajo y cebolla; Rahman (2017) encontró un mayor PV y CAL al suministrar harina de cebolla en la dosis de 0,25\%; Aji et al. (2011) consiguieron incrementos significativos del PV al incluir el ajo y cebolla en la dosis de $0,05 \%$ y $0,01 \%$ por la vía oral; Goodarzi \& Nanekarani (2014) encontraron una mayor ganancia de peso vivo, CAL y una menor CA en dietas que contenían $1 \%$ de extracto de ajos comparado con la dieta que contenían APC (Virginiamicina); Kumar et al. (2010) al incluir 0,25\% de ajo en la dieta mejoró el PV y CA en comparación al tratamiento que incluía Virginiamicina y; Karangiya et al (2016) reportó un mejor PV cuando incluyó ajo al 1\% en la dieta.

El efecto del ajo y cebolla en el rendimiento productivo varía por múltiples factores relacionados principalmente al producto. Applegate et al. (2010) al evaluar los resultados obtenidos con el uso de las plantas medicinales mencionan que la inconsistencia de los resultados se debería a la falta de un mejor conocimiento del producto, sobreventa de los posibles efectos benéficos, composición variable y falta de mayor información sobre la acción fisiológica y microbiológica in vivo.

El rendimiento productivo similar obtenido en los tres tratamientos sería por la falta de respuesta a los tratamientos, como consecuencia del bajo reto sanitario al que fueron expuestas las aves durante la crianza (Shiva et al., 2012). Las condiciones adecuadas de manejo y bioseguridad, que se consideró en la granja experimental, no permitió una mayor exposición a los patógenos. Al respecto, Bedford (2000) sostiene que los antimicrobianos que actúan en el intestino pueden no producir ningún efecto en ausencia de un desafío entérico real; a similar conclusión llegaron Moralez-Lopez et al. (2009) quienes no encontraron diferencias en el PV, CAL y CA al comparar un agente antimicrobiano (Avimicina) y probióticos (Sacharomyces serevisae) en la dieta con el grupo control y sugieren que para una evaluación eficaz de un agente antimicrobiano se requiere de un verdadero desafío bacteriano; tal como lo demuestran las investigaciones de Wati et al. (2015), Cho et al. (2014) y Amad et al. (2011) quienes mejoraron el PV y la CA con la suplementación de aditivos fitogénicos en pollos de engorde inoculados con patógenos entéricos.

La uniformidad de lote obtenida en los tres tratamientos fue elevada y con una baja variación. Un resultado similar fue obtenido por Zuidhof et al. (2015) quienes lograron una uniformidad de lote de $94,8 \%$ en pollos de engorde utilizando dietas estandarizadas y previa clasificación de los pollos (bajo, medio y alto PV); según Vasdal et al. (2019) la uniformidad en pollos de engorde es un indicador de bienestar de los animales e influye sobre el rendimiento productivo. En la investigación, la elevada uniformidad del lote lograda fue producto de las condiciones adecuadas de manejo y bioseguridad tomadas durante el proceso de crianza, lo que permitió que los tres tratamientos, expresaran su máximo potencial productivo, enmascarando el efecto antimicrobiano de los tratamientos con APC y con ajo +cebolla $(0,75 \%+$ 0,75\%) (Zuidhof, et al., 2017).

\section{Conclusiones}

La administración de harina de ajo + cebolla $(0,75 \%+$ $0,75 \%$ en la dieta tuvo rendimientos similares a los logrados por los tratamientos sin APC y con APC, con un elevado porcentaje de uniformidad de lote y podría constituir una alternativa de uso en la dieta alimenticia de los pollos de engorde.

\section{FinanCiamiento}

Investigación financiada con el Fondo Especial de Desarrollo Universitario (FEDU).

\section{REFERENCIAS}

[1] Abudabos, AM; Alyemni, AH; Dafalla, YM; UllahKhan, R. 2018. The effect of phytogenics on growth traits, blood biochemical and intestinal histology in broiler chickens exposed to Clostridium perfringens challenge. Journal of Applied Animal Research, 46(1):691-695,

https://doi.org/10.1080/09712119.2017.1383258

[2] Adibmoradi, M; Navidshad, B; Seifdavati, J; Royan, M. 2006. Effect of dietary garlic meal on histological structure of small Intestine in broiler chickens. The Journal of Poultry Science 43(4):378-383 https://doi:10.2141/jpsa.43.378

[3] Aji, SB; Ignatius, K; Ado, AA; Nuhu, JB, Abdulkarim, A; Aliyu, U; Bello, M; Adamu, M; Abubakar, H; Bukar, MM; Adama, H; Numan, P. 2011. Effects of feeding onion (Allium cepa) and garlic (Allium sativum) on some performance characteristics of broiler chickens. Research Journal of Poultry Science, 4:22-27. https://doi: 10.3923/rjpscience.2011.22.27

[4] Al-Ramamneh, D. 2018. Effect of dietary combinations of garlic and onion in broiler production. International Journal of Poultry Science, 17:147-153. https://doi: 10.3923/ijps.2018.147.153

[5] Amad, AA; Männer, K; Wendler, KR; Neumann, K; Zentek, J. 2011. Effects of a phytogenic feed additive on growth performance and ileal nutrient digestibility in broiler chickens. Poultry science, 90(12):2811-2816. 
ISSNe: 2617-9156

https://doi.org/10.3382/ps.2011-01515

[6] An, BK; Kim, JY; Oh, ST; Kang, CW; Cho, S; Kim, SK. 2015. Effects of onion extracts on growth performance, carcass characteristics and blood profiles of white mini broilers. Asian Australasian Journal of Animal Sciences 28(2);247-51. https://doi: 10.5713/ajas.14.0492

[7] Applegate, TJ; Klose, V; Steiner, T; Ganner, A; Schatzmayr, G. 2010. Probiotics and phytogenics for poultry: Myth or reality? Journal of Applied Poultry Research, 19:194-210. https://doi: 10.3382/japr.201000168

[8] (AOAC) Association of Official Agricultural Chemists. 2005. Official Methods of Analysis. 18th Edition, AOAC International, Gaithersburg, Maryland, USA. https://www.researchgate.net/publication/292783651_ AOAC_2005

[9] Bedford, M. 2000. Removal of antibiotic growth promoters from poultry diets: Implications and strategies to minimise subsequent problems. World's Poultry Science Journal, 56(4):347-365. https://doi.org/10.1079/WPS20000024

[10] Cho, JH; Kim, H; Kim, IH. 2014. Effects of phytogenic feed additive on growth performance, digestibility, blood metabolites, intestinal microbiota, meat color and relative organ weight after oral challenge with Clostridium perfringens in broilers. Livestock Science, 160:82-88. https://doi.org/10.1016/j.livsci.2013.11.006.

[11] Cobb 500. 2018a. Guía del manejo del pollo de engorde (en línea). Consultado el 09 junio 2021. Disponible en: https://cobb-500-guia-manejo.pdf (wordpress.com)

[12] Cobb 500. 2018b. Suplemento informativo sobre rendimiento y Nutrición de pollos de engorde. Consultado el 9 junio 2021. Disponible en: https://www.cobb-vantress.com/assets/Cobb-

Files/c8850fbe02/6998d7c0-12d1-11e9-9c88c51e407c53ab.pdf

[13] Corzo-Martínez, M; Nieves-Corzo, MV. 2007. Biological properties of onions and garlic. Trends in Food Science \& Technology. 12:609-62. https://doi.org/10.1016/j.tifs.2007.07.011

[14] Eid, KM; Iraquí, MM. 2014. Effect of garlic powder on growth performance and immune response for Newcastle and avian influenza virus diseases in broiler of chickens. 2nd International Conference on Biotechnology Applications In Agriculture (ICBAA). Animal Biotechnology (Poultry and Fish), 1:7-13. https://www.researchgate.net/publication/262566919

[15] Elmowalid, GA; El-Hamid, MA; El-Wahab, AA; Atta, M; El-Naser, AG; Ahmad, AA. 2019. Garlic and Ginger
Extracts Modulated Broiler Chicks Innate Immune Responses and enhanced multidrug resistant Escherichia coli O78 clearance. Comparative Immunology, Microbiology and Infectious Diseases 101334. https://doi:10.1016/j.cimid.2019.101334

[16] Goodarzi, M; Nanekarani, S. 2014. Effect of onion extract in drink water on performance and carcass traits in broiler chickens. IERI Procedia, 8:107-112. https://doi.org/10.1016/j.ieri.2014.09.018

[17] Karangiya, VK: Savsani, HH; Patil, SS; Garg, DD; Murthy, KS; Ribadiya, NK; Vekariya, S. J. 2016. Effect of dietary supplementation of garlic, ginger and their combination on feed intake, growth performance and economics in commercial broilers. Veterinary World, 9(3):245-50. https:// doi: 10.14202/vetworld.2016.245250

[18] Kumar, S; Sharadamma, KC; Radhakrishna, PM. 2010. Effects of a garlic active based growth promoter on growth performance and specific pathogenic intestinal. Microbial counts of broiler chicks. International Journal of Poultry Science 9(3):244-246. https://doi: 10.3923/ijps.2010.244.246

[19] Morales-López, R; Auclair, E; García, F; EsteveGarcía, E; Brufau, J. 2009. Use of yeast cell walls; $\beta-1$, 3/1, 6-glucans; and mannoproteins in broiler chicken diets. Poultry Science, 88(3),601-607. https://doi.org/10.3382/ps.2008-00298

[20] Mulugeta, M. 2018. Review on efficacy of garlic and onion on performances, blood profile and health status of broiler chickens. Global Journal of Science Frontier Research, $18 \quad$ (6):36-46. https://globaljournals.org/GJSFR_Volume18/4Review-on-Efficacy-of-Garlic.pdf

[21] Ojediran, TK; Fasola, MO; Oladele, TO; Onipede, TL; Emiola, IA. 2017. Growth performance, flock uniformity and economic indices of broiler chickens fed low crude protein diets supplemented with lysine. Archivos de Zootecnia, 66(256):543-550.

[22] https://www.redalyc.org/articulo.oa?id=49553571010

[23] Rahman, S; Khan, S; Chand, N; Sadique, U; Khan, RU. 2017. In vivo effects of Allium cepa L. on the selected gut microflora and intestinal histomorphology in broiler. Acta Histochemica, 119(5):446-450. http://dx.doi.org/10.1016/j.acthis.2017.04.004.

[24] Roskam, JL; Oude, AGJM; Saatkamp, HW. 2020. The relation between technical farm performance and antimicrobial use of broiler farms. Poultry Science, 99 (3):1349-1356.

https://doi.org/10.1016/j.psj.2019.10.054 
ISSNe: 2617-9156

[25] Rostagno, HS; Texeira, LF; Hannas, MI; Kazue, N; Perazzo, FG; Saraiva, A; Texeira, ML; Borges, P; de Oliveira, FR; de Toledo, SL; de Oliveira, C. 2017. Tablas brasileñas para aves y cerdos. Composición de los alimentos y requerimientos nutricionales. 4ta ed. Universidad Federal de Vicosa, Brazil. https://eliasnutri.files.wordpress.com/2018/09/tablasbrasilec3b1as-aves-y-cerdos-cuarta-edicion-201711.pdf

[26] Salem, WM; El-hamed, DM; Sayed, WF; Elamary, RB. 2017. Alterations in virulence and antibiotic resistant genes of multidrug-resistant Salmonella serovars isolated from poultry: The bactericidal efficacy of Allium sativum. Microbial Pathogenesis, 108:91-100. https://doi.org/10.1016/j.micpath.2017.05.008

[27] Sharma, P; Kumar, S; Goswami, P; Sangwan, V; Singh, R. 2014. Antibiotic resistance among commercially available probiotics. Food Research International, 57:176-195. https://doi.org/10.1016/j.foodres.2014.01.025

[28] Shiva, C; Bernal, S; Sauvain, M; Caldas, J; Kalinowski, J; Falcón, N; Rojas, R. 2012. Evaluación del aceite esencial de orégano (Origanum vulgare) y extracto deshidratado de jengibre (Zingiber officinale) como potenciales promotores de crecimiento en pollos de engorde. Revista de Investigaciones Veterinarias del Perú, 23(2):160-170 https://doi.org/10.15381/rivep.v23i2.896

[29] Sugiharto, S; Ranjitkar, S. 2019. Recent advances in fermented feeds towards improved broiler chicken performance, gastrointestinal tract microecology and immune responses: a review. Animal Nutrition 5:1-10. https://doi.org/10.1016/j.aninu.2018.11.001

[30] Vasdal, GE; Granquist, EG., Skjerve, E; De Jong, IC; Berg, C; Michel, V; Moe, RO. 2019. Associations between carcass weight uniformity and production measures on farm and at slaughter in commercial broiler flocks. Poultry Science, 98(10):4261-4268. https://doi.org/10.3382/ps/pez252

[31] Wati, T; Ghosh, T; Syed, B; Haldar, S. 2015. Comparative efficacy of a phytogenic feed additive and an antibiotic growth promoter on production performance, caecal microbial population and humoral immune response of broiler chickens inoculated with enteric pathogens. Animal Nutrition, 1:213 - 219 https://doi.org/10.1016/j.aninu.2015.08.003.

[32] Zuidhof, MJ; Holm, DE; Renema, RA; Jalalh, MA; Robinson, FE. 2015. Effects of broiler breeder management on pullet body weight and carcass uniformity. Poultry Science, 94(6):1389-1397. https://doi.org/10.3382/ps/pev064
[33] Zuidhof, MJ; Fedorak, MV; Quellette, CA; Wenger, II. 2017. Precision feeding: Innovative management of broiler breeder feed intake and flock uniformity. Poultry Science, 96(7):2254-2263. https://doi:10.3382/ps/pex013 